Vertragsfreiheit unvereinbar ${ }^{97}$. Mit der Privatautonomie ist es beispielsweise kaum zu vereinbaren, wenn ein Gastwirt aufgrund europäischen Produkthaftungsrechts für unverschuldet mit Hepatitis infizierte Speisen bis zu einem Betrag von $€ 70$ Mio. zwingend unbeschränkbar haften soll. Die immer weiter ausgreifende Anordnung von Kontrahierungszwängen beschränkt die Abschlussfreiheit in schwer vertretbarer Weise. Auch darf die Einräumung von Widerrufsrechten nicht dazu führen, dass in weiten Bereichen des Vertragsrechts dauernde Ungewissheit über die Beständigkeit des Vertragsschlusses besteht. Das europäische Richtlinienrecht schenkt der Vertragsfreiheit letztlich nicht immer die gebotene Aufmerksamkeit.

\section{Movement from Contract to Status im europäischen Vertragsrecht}

Wenn man davon ausgeht, dass das europäische Richtlinienrecht mit der Anordnung zwingenden Rechts, weitgehender Inhaltskontrolle und Einschränkungen der Abschlussfreiheit durch Widerrufsrechte und Abschlussregeln der Vertragsfreiheit grundsätzlich entspricht, ist zu fragen, ob das europäische Vertragsrecht mit der zunehmenden Einschränkung der Privatautonomie, die einem paternalistischen Grundverständnis entspricht, auf dem richtigen Weg ist. Wenn man sich die historischen Wurzeln der Vertragsfreiheit und ihre wichtigen Funktionen in einer demokratisch verfassten Rechtsordnung vergegenwärtigt, sind hier durchaus Zweifel angebracht. Es dürfte letztlich kein Zufall sein, dass in den USA - einer der ältesten und stabilsten Demokratien der Welt - die liberal verstandene Vertragsfreiheit einen hohen Stellenwert hat. Das US-amerikanische Vertragsrecht war und ist damit bislang im Großen und Ganzen nicht schlecht beraten. Die EU befindet sich dagegen derzeit, wenn die Zeichen nicht trügen, eher auf dem Weg zur zentral regulierten Privatrechtsordnung mit paternalistisch ausgeformter Vertragsfreiheit. In Ansätzen zeigt sich ein europäisches „Movement from Contract to Status“, das bei ungehinderter Fortentwicklung $\mathrm{zu}$ einer Lähmung des Privatrechts führen könnte. Auch in historischer Perspektive liegt darin kein unbedingter Fortschritt. Es bleibt zu hoffen, dass der europäische Gesetzgeber und der EuGH die Zeichen der Zeit erkennen und sich bei der weiteren Ausgestaltung des europäischen Privatrechts die notwendige Berücksichtigung der

97 Näher Bruns (Fn. 50), S. 275 f. liberal verstandenen Vertragsfreiheit verordnen. Diese Hoffnung gilt auch zukünftigem Handeln des deutschen Gesetzgebers - nicht nur bei der Umsetzung europäischer Vorhaben - und der Judikatur von $B \operatorname{VerfG}$ und $B G H$.

\section{Zusammenfassung}

1. Grundsätzlich stehen sich zwei unterschiedliche Grundmodelle der Vertragsfreiheit gegenüber: ein liberales und ein paternalistisches. Die historischen Wurzeln der Privatautonomie liegen in der Aufklärung und im Liberalismus und damit in der eigenverantwortlich bestimmten Vertragsgerechtigkeit.

2. Im deutschen Vertragsrecht zeigt sich eine Tendenz zur paternalistischen Ausformung der Vertragsfreiheit durch zunehmende Beschränkungen von Abschluss- und Inhaltsfreiheit. Die Regelung der Abschlusszwänge im neuen Allgemeinen Gleichbehandlungsgesetz ist in puncto Beweislast systemwidrig und in ihrer Vereinbarkeit mit der Vertragsfreiheit fragwürdig.

3. Die Vertragsfreiheit, verstanden als Freiheit zu eigenverantwortlicher Gestaltung privatrechtlicher Rechtsbeziehungen, ist Garant von Demokratie, von zukunftsfähiger Innovationskraft und Stabilität der Rechtsordnung.

4. Im angloamerikanischen Vertragsrecht hat die Vertragsfreiheit historisch bedingt bis heute einen hohen Stellenwert im materiellen Recht und Verfahrensrecht. Inhaltsund Abschlusskontrolle von Verträgen sind weit weniger stark ausgeprägt als im europäischen Vertragsrecht.

5. Das europäische Vertragsrecht zeigt eine deutliche Tendenz von der liberal verstandenen Vertragsfreiheit hin zu einem paternalistischen Verständnis der Privatautonomie, das der eigenverantwortlichen Vertragsgestaltung durch die Parteien immer weniger Spielräume lässt. Die europäische primärrechtliche Grundgewährleistung der Vertragsfreiheit erfährt dabei nicht immer die notwendige Gewichtung.

6. Für das europäische Privatrecht kann man zugespitzt von einem „Movement from Contract to Status“ sprechen. Diese Abkehr vom überkommenen liberalen Grundverständnis der Privatautonomie bedeutet weder historisch noch rechtskulturell einen Fortschritt. Europäische und nationale Rechtsetzung und Rechtsprechung sollten der Versuchung weiterer regulierender Beschneidung der Vertragsfreiheit widerstehen.

Privatdozent Dr. Matthias Rossi, Berlin*

\title{
Verschuldungsautonomie und Entschuldungsverantwortung
}

\author{
Interimsbedingungen der Bewältigung von Haushaltsnotlagen
}

Mit Urteil vom 19. 10. 2006** hat das BVerfG klargestellt, dass ein Bundesland nur im Falle eines „bundesstaatlichen Notstands" Anspruch auf Bundeshilfen zur Bewältigung extremer Haushaltsnotlagen hat, im Übrigen aber allein für seine Entschuldung verantwortlich ist. Die nunmehr verstärkt am Leitbild von Autonomie und Eigenverantwortlichkeit der

* Dr. Matthias Rossi ist Privatdozent der Humboldt-Universität zu Berlin und vertritt im Sommersemester 2007 einen Lehrstuhl an der Universität Karlsruhe.
Bundesländer ausgelegten verfassungsrechtlichen Vorgaben sind solange von allen hilfebegehrenden Ländern als verbindlicher Maßstab zu beachten, bis der Bund und die Länder sich - etwa im Rahmen der Föderalismusreform II auf neue oder ergänzende Regelungen zur Bewältigung von Haushaltnotlagen verständigt haben. ${ }^{* *}$

** $\quad B \operatorname{Verf} G E$ 116, 327. Das Urteil ist auch zu finden unter bttp://www. bundesverfassungsgericht.de/entscheidungen/fs20061019_2bvf000303.html. 


\section{Verschärfte Voraussetzungen des Anspruchs auf Sanierungszuweisungen}

Das BVerfG knüpft in seinem „Berlin-Urteil“ an frühere Entscheidungen an, ${ }^{1}$ insbesondere auch an sein Urteil aus dem Jahre 1992, in dessen Folge das Saarland und Bremen zusätzliche Bundesmittel erhalten haben. ${ }^{2}$ Es betont zugleich, dass die in diesen Entscheidungen aufgestellten Maßstäbe „nicht mehr ohne weiteres fortzuschreiben, sondern verschärfend zu ergänzen sind. “ 3 Die relativ moderate Bezeichnung als „verschärfende Ergänzung“ der bisherigen Rechtsprechung verstellt den Blick für einen deutlichen Kurswechsel: Leitmaxime ist nicht mehr primär das solidarische, aus dem Bundesstaatsprinzip abgeleitete „bündische Prinzip des Einstehens füreinander“, ${ }^{4}$ sondern vor allem die Eigenverantwortung der Bundesländer.

\section{Eigenverantwortung der Bundesländer}

Dass das Urteil die Eigenverantwortung der Bundesländer mehr denn ihre Solidarität untereinander betont, wird nicht nur am Ergebnis sichtbar. Wenn das $B \operatorname{VerfG}$ in seiner Begründung hervorhebt, dass ein Land auf Grund seiner verfassungsrechtlich in Art.109 Abs.1 GG verbürgten Haushaltsautonomie grundsätzlich alle haushaltswirtschaftlichen Maßnahmen selbst zu verantworten hat, ${ }^{5}$ dann kommt darin zum Ausdruck, dass der grundsätzlichen Verschuldungsautonomie auch die regelmäßige Entschuldungsverantwortung eines Landes entspricht. Diese Entschuldungsverantwortung kann und darf nicht bzw. nur unter besonderen, nahezu nicht erfüllbaren Voraussetzungen durch Bundesergänzungszuweisungen nach Art. 107 Abs. 3 S. 2 GG unterlaufen werden. Diese dienen nach den deutlichen Worten des BVerfG nämlich grundsätzlich nicht dazu, „finanziellen Schwächen abzuhelfen, die eine unmittelbare und voraussehbare Folge von politischen Entscheidungen sind, die von einem Land in Wahrnehmung seiner Aufgaben selbst getroffen sind." Vielmehr bringen es Eigenständigkeit und politische Autonomie mit sich, wie das BVerfG unter Bezugnahme früherer Entscheidungen ausführt, „dass die Länder für die haushaltspolitischen Folgen solcher Entscheidungen selbst einzustehen haben.“6

Diesem Verständnis entspricht es, wenn das $B \operatorname{Verf} G \mathrm{da}$ rauf hinweist, dass „die so genannte Verschuldungsfalle [...] nicht überraschend von heute auf morgen zu[schnappt] ". ${ }^{7}$ So zutreffend diese Beobachtung in tatsächlicher Hinsicht ist, so sehr hat es das $B \operatorname{VerfG}$ an dieser Stelle versäumt, in rechtlicher Hinsicht auf den Zusammenhang mit den landesverfassungsrechtlichen Verschuldungsgrenzen hinzuweisen. Denn eine Verschuldung kommt in der Höhe, in der sich ein Land wegen der verursachten Zinslasten in einer Verschuldungsfalle fühlt, regelmäßig nur zu Stande, wenn über Jahre hinweg die landesverfassungsrechtlichen Verschuldungsgrenzen missachtet, wenn also über mehrere Jahre verfassungswidrige Haushaltsgesetze verabschiedet und vollzogen werden. Mit anderen Worten und deutlicher: Bei Beachtung der verfassungsrechtlichen Verschuldungsgrenzen ist es so

1 Vgl. BVerfGE 72, 330; 79, 311; 86, $148=\mathrm{JZ}$ 1962, 962 m. Anm. Arndt; 91,$186 ; 101,158$.

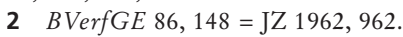

$3 \quad B \operatorname{VerfGE} 116,327,389$.

4 Vgl. $B \operatorname{VerfGE} 72,330$ (386); 86, 148 (214f.) = JZ 1962, 962; 101, 158 (221).

$5 B \operatorname{VerfGE} 116,327,399$

$6 B \operatorname{VerfGE} 116,327,385$

7 BVerfGE 116, 327, 391 gut wie ausgeschlossen, dass ein Land in eine Situation gerät, aus der es sich nur noch mit Hilfe des Bundes und der anderen Länder glaubt befreien zu können. Eine erste und wichtige Konsequenz der Betonung der Eigenverantwortlichkeit der Länder einschließlich ihrer grundsätzlichen Verschuldungsautonomie ist mithin, dass die verfassungsrechtlichen (und gemeinschaftsrechtlichen) Verschuldungsgrenzen strikt einzuhalten sind, um das Entstehen einer übermäßigen Haushaltsnotlage zu verhindern. Dies verlangt unter Umständen auch neue Klagebefugnisse, um die Beachtung der Verschuldungsgrenzen aus einem primär politischen in ein rein rechtliches Kontroll- und Sanktionssystem zu überführen. ${ }^{8}$

Im Übrigen ist die Betonung der Eigenverantwortlichkeit der Länder aus mindestens drei Gründen zu begrüßen: Erstens ist nüchtern festzustellen, dass die auf dem „bündischen Prinzip des Einstehens füreinander" basierenden Sanierungszuweisungen des Bundes an Bremen und das Saarland nicht den erwünschten Erfolg gebracht haben. Obwohl in der Zeit von 1994 bis 2004 rund 15 Mrd. Euro an die beiden notleidenden Bundesländer gezahlt wurden, befinden sie sich nach eigener Wahrnehmung noch immer in einer extremen Haushaltsnotlage, klagen deshalb erneut auf Sanierungshilfen des Bundes und haben ihre entsprechenden Anträge auch nach dem neue Maßstäbe setzenden „Berlin-Urteil“ des $B \operatorname{VerfG}$ nicht zurückgenommen. Die solidarische Unterstützung hat die betroffenen Länder nicht aus ihrer Notlage befreit und sich deshalb nicht bewährt.

Nicht zuletzt deshalb wird zweitens vor allem aus finanzwissenschaftlicher, daneben aber auch aus rechtlicher und zum Teil auch aus politischer Sicht seit langem gefordert, die politischen Anreize zur Vermeidung von Haushaltsnotlagen zu stärken. ${ }^{9}$ Eine auf dem Gedanken der Solidargemeinschaft beruhende Einstandspflicht des Bundes für notleidende Länder mit der Möglichkeit des bail-out, also der vollständigen oder jedenfalls teilweisen Schuldenübernahme durch die Gemeinschaft von Bund und anderen Ländern, verhindert insbesondere für Länder in oder am Rande einer extremen Haushaltsnotlage die Motivation, sich aus eigenen Kräften zu entschulden. Umgekehrt und zugleich werden auch in den finanzkräftigeren Ländern die Anreize zu einer konsolidierenden und konsolidierten Haushaltspolitik geschwächt, weil ein Großteil ihrer Bemühungen über den Haushaltsausgleich nur dem Länderdurchschnitt und deshalb eben auch anderen Ländern zu Gute kommt. Ein an der Eigenverantwortung der Länder ausgerichtetes Verständnis der Entschuldungsautonomie mit der weitgehend reduzierten, wohl nur noch theoretisch bestehenden Möglichkeit des bail-out ist insofern ein wichtiges Signal an alle Länder, ihre Haushalte (und d.h. weitgehend ihre Ausgaben) alleine in den Griff bekommen zu müssen. Wollen sie politischen und d.h. finanziellen Handlungsspielraum behalten oder sogar gewinnen, müssen sie ihre Zins- und Tilgungslasten aus eigener Kraft durch eine Reduzierung der Verschuldung verringern. Im Übrigen geht mit der Anreizwirkung für die politischen Akteure in den Ländern zugleich auch eine Signalwirkung für deren Bürger einher. Denn mögen sie die Ursachen für die Verschuldung unter Umständen auch weiterhin außerhalb ihrer Landesgrenzen suchen, muss ihnen nach dem Berlin-Urteil klar sein, dass für die Entschuldung allein das eigene Land verantwortlich ist. Sie werden deshalb künftig eine strikte Konsolidierungspolitik vielleicht

8 Siehe unten III 4 a).

9 Vgl. die einzelnen Beiträge in Konrad/Jochimsen (Hrsg.), Finanzkrise im Bundesstaat, 2006. 
stärker bei ihrer Wahlentscheidung honorieren und damit einen weiteren und entscheidenden Anreiz für die politischen Akteure schaffen. ${ }^{10}$

Drittens schließlich muss betont werden, dass die Neuausrichtung an der Eigenverantwortlichkeit der Bundesländer keine ureigene oder gar willkürliche, vom politischen Willen der gesetzgebenden Körperschaften abgekoppelte Entscheidung von acht nur mittelbar demokratisch legitimierten Richterinnen und Richtern des BVerfG ist. Vielmehr hat der zweite Senat den Wandel vom eher kooperativen zum auch kompetitiven, ${ }^{11}$ jedenfalls aber die Eigenständigkeit der einzelnen Glieder betonenden Bundesstaat nur nachvollzogen, den der verfassungsändernde Gesetzgeber bereits mit der so genannten Verfassungsreform von 199412 eingeleitet und mit der so genannten Föderalismusreform I jüngst fortgesetzt hat. Ziel dieser Verfassungsänderungen war und ist es unter anderem, die Kompetenz- und damit die Verantwortungsbereiche von Bund und Ländern deutlicher zu trennen und den Ländern mehr substantielle Zuständigkeiten zuzuweisen. Speziell im Finanzverfassungsrecht wird der politische Wille, die Eigenverantwortung der Bundesländer zu stärken, an dem bereits in der Föderalismusreform I aufgenommenen Art. 104 Abs.6 S. 3 GG deutlich, nach dem die durch die Verletzung von internationalem und vor allem supranationalem Recht hervorgerufenen finanziellen Lasten nicht allein nach dem "Solidarprinzip“, sondern auch unter Berücksichtigung des „Verursacherprinzips" auf Bund und Länder verteilt werden. Insofern antizipiert die noch auf Grundlage der alten Rechtsordnung zur binnenstaatlichen Verteilung europarechtlicher Anlastungen ergangene Entscheidung des BVerfG vom 19.10. 2006, die maßgeblich auf die Verursachung - wohlgemerkt nicht auf das Verschulden - der einzelnen Länder und des Bundes abstellt $^{13}$ und damit ebenfalls deren Eigenverantwortung betont, die Entscheidung des verfassungsändernden Gesetzgebers.

\section{Sanierungszuweisungen im System des Finanzausgleichs}

Entsprechend der verstärkten Ausrichtung an der Eigenverantwortlichkeit der Bundesländer betont das $B V \operatorname{erf} G$ einmal mehr und deutlicher als früher, dass Sonderzuweisungen zum Zwecke der Haushaltssanierung einen Fremdkörper im System des Finanzausgleichs darstellen. Nicht zuletzt deshalb kommen solche Zuweisungen nur als ultima ratio in Betracht. Damit hat das BVerfG den Ausnahmecharakter von Sanierungszuweisungen, den es schon in seinen vergangenen Entscheidungen hervorgehoben hat, ${ }^{14}$ begrifflich auf den Punkt gebracht. Das „strenge Ultima-ratio-Prinzip“ kommt insbesondere in den hohen materiellen und formellen Voraussetzungen zum Ausdruck, unter denen Sanierungszuweisungen künftig nur noch geleistet werden dürfen. $\mathrm{Zu}$ sätzlich zum relativen, auf den Länderdurchschnitt abstellenden Maßstab kommt ein absoluter Maßstab hinzu, der

10 Freilich hat das Anreizdilemma seine Ursache auch in der auf Zeit beschränkten Herrschaft, die einer Demokratie wesensimmanent ist.

11 Zum Wettbewerbsföderalismus vgl. etwa Schatz/van Ooyen/Werthes, Wettbewerbsföderalismus. Aufstieg und Fall eines politischen Streitbegriffs, 2000, passim.

12 Vgl. hierzu Kloepfer/Lang, Verfassungsänderung statt Verfassungsreform, 2. Aufl., 1996, passim.

$13 B \operatorname{VerfGE} 116,327,385$.

$14 B \operatorname{VerfGE} 72,330$ (405); 101, 158 (225). insbesondere dann virulent wird, wenn schon der Länderdurchschnitt eine Haushaltsnotlage indiziert. ${ }^{15}$

Zweierlei ist freilich für das richtige Verständnis von Sanierungszuweisungen im geltenden System des Finanzausgleichs zu betonen: Erstens ist zu vergegenwärtigen, dass Sanierungszuweisungen primär deshalb als Fremdkörper im geltenden System des Finanzausgleichs eingestuft werden, weil dieses System kein speziell auf Haushaltssanierungen zugeschnittenes Instrument bereithält. Das BVerfG stellt deshalb weder die rechtliche Zulässigkeit noch die politische Sinnhaftigkeit von Finanztransfers zum Zwecke der Haushaltssanierung als solche in Frage, sondern hat nur geprüft, ob die vom Finanzverfassungsrecht explizit vorgesehenen Instrumente des Finanzausgleichs, namentlich die von Art. 107 Abs. 3 S. 2 GG vorgesehenen Bundesergänzungszuweisungen, ausnahmsweise auch zweckwidrig oder jedenfalls zweckentfremdend für Sanierungszuweisungen verwendet werden können. Der durch „ein strenges Ultima-ratioPrinzip“ zu sichernde Ausnahmecharakter hat seinen Grund also vor allem in dem Missbrauch von Finanzausgleichsinstrumenten zu dem von diesen nicht intendierten Zweck der Haushaltssanierung. Daraus folgt de constitutione lata, dass die unterschiedliche Finanzkraft der Bundesländer auf den anderen Stufen des Finanzausgleichs angemessen berücksichtigt werden kann und muss, wie das $B \operatorname{VerfG} \mathrm{zu}$ Recht betont. Mit Blick auf die Hauptstadtfunktion Berlins ist dabei insbesondere auch Art. 106 Abs. 8 GG zu berücksichtigen. Und de constitutione ferenda steht es dem verfassungsändernden Gesetzgeber frei, ein gesondertes Instrument zur Bewältigung von Haushaltsnotlagen in einzelnen Bundesländern zu schaffen.

Hervorzuheben ist zweitens aber auch, dass das $B \operatorname{Verf} G$ für den Fall, dass die formellen und materiellen Voraussetzungen tatsächlich gegeben sind, nicht nur von der verfassungsrechtlichen Zulässigkeit, sondern auch von der verfassungsrechtlichen Pflicht zur Leistung von Sanierungshilfen ausgeht: „Weil und soweit Situationen eintreten, in denen die verfassungsrechtlich gebotene Handlungsfähigkeit eines Landes nicht anders aufrecht zu erhalten ist, ist bundesstaatliche Hilfeleistung durch Mittel zur Sanierung als ultima ratio erlaubt und dann auch geboten."16 Insofern kommt in diesen Fällen eben doch das bündische Prinzip des Einstehens füreinander zum Tragen, dessen Anwendungsbereich durch die enorm hohen materiellen und formellen Voraussetzungen freilich schon theoretisch sehr schmal wird und praktisch gen Null tendieren dürfte. ${ }^{17}$

\section{Voraussetzungen von Sanierungszuweisungen}

Im geltenden Finanzverfassungsrecht stehen Sonderzuweisungen zum Zwecke der Haushaltssanierung künftig unter der materiellen Voraussetzung eines bundesstaatlichen Notstands, der in formeller Hinsicht vom hilfebegehrenden Land dargelegt und begründet werden muss.

$15 \quad B \operatorname{VerfGE} 116,327,387$.

16 Hervorhebung durch den Autor.

17 Allerdings genügt den kreditgebenden Gläubigern wohl schon die nur theoretische Möglichkeit des bail-out, und sei sie noch so gering, wie die kaum zu spürenden Reaktionen der Rating-Agenturen auf das Berlin-Urteil gezeigt haben: Sie schätzen die Kreditwürdigkeit der stark verschuldeten Bundesländer trotz der Betonung von deren Entschuldungsverantwortung ganz überwiegend weiterhin mit dem höchsten AAA-Standard ein. Vgl. hierzu die Beiträge von Stadler und Strasser, in: Konrad/Jochimsen (Hrsg.), Der Föderalstaat nach dem Berlinurteil, 2007 (im Erscheinen). 


\section{a) Bundesstaatlicher Notstand}

Begrifflich kristallisiert sich das Neue des Berlin-Urteils in dem Terminus „bundesstaatlicher Notstand“. Ohne diesen Begriff näher zu definieren, erhebt das $B V \operatorname{Verf} G$ ihn zu der materiellen Voraussetzung, unter der ausnahmsweise Sanierungshilfen des Bundes zulässig (und geboten) sind. Dabei bleibt das Verhältnis zwischen dem ehemals und noch immer verwendeten Begriff der extremen Haushaltsnotlage und dem neu geschöpften Begriff „bundesstaatlicher Notstand“ unklar. Die fehlende Definition bzw. unzureichende Umschreibung des Begriffs „bundesstaatlicher Notstand“ ist sicherlich kein Zufall. Vielmehr ist davon auszugehen, dass das $B V \operatorname{ver} G$ den Begriff aus mehreren Gründen bewusst im Unklaren gelassen hat.

Zum einen behält das Gericht auf diese Weise einen Spielraum, um auf künftige Klagen unter Berücksichtigung etwaiger besonderer Umstände eventuell doch das solidarische Prinzip gegenüber dem Prinzip der Eigenverantwortung stärker betonen zu können, ohne dadurch in einen (offenen) Widerspruch mit der eigenen Rechtsprechung zu geraten. Zwar ist mit Blick auf die noch anhängigen Normenkontrollanträge Bremens und des Saarlandes nicht damit zu rechnen, dass das $B \operatorname{Verf} G$ bereits in diesen Fällen von einem bundesstaatlichen Notstand ausgeht. Doch kann und will das $B \operatorname{Verf} G$ offensichtlich nicht ausschließen, dass es in der $\mathrm{Zu}$ kunft einmal einen Anspruch auf Sanierungszuweisungen bejahen muss, um einen bundesstaatlichen Notstand abzuwehren.

Zum anderen entspricht die fehlende Definition des Begriffs „bundesstaatlicher Notstand“ der generellen Zurückhaltung des Urteils gegenüber der politischen Gestaltungsfreiheit der gesetzgebenden Körperschaften. Das Urteil signalisiert sowohl im Ergebnis als auch in seiner Begründung deutlich, dass das $B \operatorname{Verf} G$ sich nicht für zuständig hält, die grundlegenden Fragen des föderalen Finanzausgleichs verbindlich zu beantworten. Vielmehr spielt es den Ball offen an die Politik zurück. Dies ist insofern verständlich, als es sowohl unter dem Gesichtspunkt demokratischer Legitimation als auch unter dem Aspekt funktionaler Gewaltenteilung Aufgabe der legislativen Gewalt ist, die Voraussetzungen und Bedingungen festzulegen, unter denen an die Stelle des mit der Eigenstaatlichkeit der Länder korrespondierenden Prinzips der Eigenverantwortung das Prinzip der Solidarverantwortung tritt. Die besondere Elastizität des Bundesstaatsprinzips ${ }^{18}$ gibt jedenfalls dem verfassungsändernden und in Grenzen dem einfachen Gesetzgeber insoweit auch die Freiheit, den Begriff des bundesstaatlichen Notstands mit Inhalt zu füllen.

Schließlich mag das BVerfG den Begriff aus einem dritten Grunde unscharf gelassen haben, der unter Anreiz-Gesichtspunkten zu begrüßen ist. Denn indem und weil der Begriff nicht definiert wird, kann eben auch nicht exakt ausgerechnet und vorhergesehen werden, ob und wann ein bundesstaatlicher Notstand vorliegt. Der einerseits eigene Not indizierende, andererseits aber auch fremde Hilfe versprechende Begriff des bundesstaatlichen Notstands entzieht sich somit dem Kalkül politischer Handlungsalternativen. In der (vermeintlichen) Wahl stark verschuldeter Länder, ihre Haushalte entweder durch eigene Anstrengungen zu konsolidieren oder aber, auf fremde Hilfe hoffend, in den bundesstaatlichen Notstand zu steuern, ist als letzte Alternative mangels Klarheit des Ziels derart riskant, dass doch nur die erste Alternative bleibt. Die Unschärfe des Begriffs trägt so-

18 Selmer VVdStRL 52 (1993), 10 (22). mit entscheidend dazu bei, dass es im geltenden Finanzverfassungsrecht grundsätzlich keine Bail-out-Möglichkeit gibt, sondern die Entschuldungsverantwortung allein bei den Bundesländern bleibt.

Versucht man trotz seiner wohl bewussten Offenheit eine Annäherung an den Begriff „bundesstaatlicher Notstand“, so wird deutlich, dass das $B \operatorname{VerfG}$ mit ihm nicht nur verschärfte Voraussetzungen einer extremen Haushaltsnotlage beschrieben, sondern einen völlig anderen und neuen Maßstab aufgestellt hat. Dies folgt zwar noch nicht aus dem substantivischen Wandel von einer „Notlage“ zu einem „Notstand“. Insbesondere gibt es keinen Hinweis in der Entscheidung und auch keinen erkennbaren sonstigen Anlass, den Begriff unter Rückgriff auf Art.81 GG bestimmen zu müssen. Doch seinem Wortlaut nach ist der Begriff eben nicht mehr nur auf den Haushalt beschränkt, sondern von dieser Umschreibung befreit und statt dessen um die Bezugnahme des Bundesstaates erweitert. Gerade ausweislich seiner adjektivischen Umschreibung verlangt der Begriff deshalb in materieller Hinsicht, dass der Notstand nicht nur auf das hilfebegehrende Land begrenzt ist, sondern solche Ausmaße erreicht, dass über die Landesgrenzen hinweg auch das gesamte bundesstaatliche Gefüge gefährdet ist. Entscheidend sind somit nicht allein bestimmte finanzwissenschaftliche, auf das notleidende Land bezogene Eckdaten, sondern ist der Umstand, dass ein Bundesland die ihm verfassungsrechtlich obliegenden Aufgaben in einer Weise nicht erfüllen kann, dass auch die Aufgabenerfüllung in den anderen Ländern und im Bund in Mitleidenschaft gerät. Die Bezugsgröße für die Einstandspflicht des Bundes ist somit nicht mehr nur das notleidende Land, sondern ist die auf die Gesamtheit von Bund und Ländern bezogene Ordnung. Dies wird auch daran deutlich, dass das $B \operatorname{Verf} G$ die bundesstaatlichen Einstandspflichten im Interesse aller Bundesbürger und nicht nur im Interesse der jeweils betroffenen Landesbürger sieht. ${ }^{19}$ Weder der Bund noch die anderen Länder wollen, können und dürfen sich ein Land leisten, in dem etwa essentielle Sicherheits- oder bspw. auch „nur“ Bildungsaufgaben mangels finanzieller Mittel nicht mehr wahrgenommen werden können. Sie widersprächen ansonsten dem gemeinsamen Interesse aller Bundesbürger.

In formeller Hinsicht könnte und muss der solcher Art verstandene Begriff implizieren, dass der Notstand nicht von einem Land allein festgestellt werden kann, sondern dies einem Gremium unter Beteiligung des Bundes und der anderen Länder bzw. bis zu dessen Bildung dem $B$ VerfG obliegen muss. Anknüpfen lässt sich insofern an den bereits existierenden Finanzplanungsrat oder besser noch an Vorschläge für einen Sanierungsrat. ${ }^{20}$

Nur ein solches Verständnis vom Begriff des bundesstaatlichen Notstands trägt übrigens der Erkenntnis Rechnung, dass - jedenfalls in Bezug auf das Haushaltsrecht nicht an dem bloß zweigliedrigen Bundesstaatsbegriff festgehalten werden kann, ${ }^{21}$ nach dem es neben der Ebene des Bundes und der der Länder keine dritte, den Bund und die Länder umfassende Ebene gibt. Denn die Haushaltswirtschaften von Bund und Ländern sind bei aller rechtlichen, durch Art. 109 Abs. 1 GG verfassungskräftig geschützten Eigenständigkeit faktisch so miteinander verzahnt, dass sich

$19 B \operatorname{VerfG} E$ 116, 327, 392.

20 Vgl. Wissenschaftlicher Beirat beim BMF, Haushaltskrisen im Bundesstaat, Bd.78 der Schriftenreihe des BMF; Rossi/Schuppert ZPR 2006, 8 (9); Fiedler u.a., Die finanzielle Zukunft Berlins - Vorschlag eines konditionierten Sanierungsverfahrens für die Hauptstadt, Studie der Hertie-School of Governance, 2006, S. $181 \mathrm{ff}$.

21 Vgl. Rossi DVBl. 2005, 269 (271f.). 
die Haushaltskrise in einem Bundesland über die schwindende Fähigkeit zur Aufgabenerfüllung auch auf die gemeinsamen Interessen des Bundes und der anderen Ländern auswirkt. Dass das BVerfG jedenfalls in Ansätzen auf die Interessen der Bundesbürger abstellt, zeigt deutlich das die Zweiteilung auf- und die Gesamtheit hervorhebende Verständnis des Bundesstaats.

\section{b) Darlegungs- und Begründungslasten}

Im Bewusstsein der Unbestimmtheit des Begriffs „bundesstaatlicher Notstand" legt das BVerfG dem hilfebegehrenden Land in formeller Hinsicht erhebliche Darlegungs- und Begründungslasten auf. Es knüpft damit erkennbar an seine Rechtsprechung zum Verschuldungsverbot in Art.115 GG an. Sollen dort die Darlegungs- und Begründungslasten dazu beitragen, „die Inanspruchnahme der Ausnahmebefugnis zu erhöhter Kreditaufnahme [...] - trotz des Fehlens eindeutiger materiell-rechtlicher Vorgaben - auf Ausnahmefälle zu beschränken und so ihren Ausnahmecharakter zu sichern“,22 sollen sie hier dem „Problem notwendiger, politischer aber wohl immer umstrittener Bewertung noch vorhandener Handlungsmöglichkeiten des Landes" angemessen und zumutbar begegnen. ${ }^{23}$ In beiden Fällen werden die einer politischen Bewertung recht offenen materiellen Voraussetzungen somit durch formelle Anforderungen kompensiert. $^{24}$

Die Bedeutung dieser verfahrensmäßigen Darlegungsund Begründungslasten ist nicht zu unterschätzen. So wie allein sie - erfolgreich, wie zuletzt die Entscheidung des VerfGH Berlin gezeigt hat ${ }^{25}$ - den Ausnahmecharakter der verfassungsrechtlichen Verschuldungsbegrenzung sichern, indem sie sowohl dem Haushaltsgesetzgeber selbst als auch der Öffentlichkeit das Überschreiten der grundsätzlichen Verschuldungsgrenze vor Augen führen, so garantieren nur sie das Ultima-ratio-Prinzip von Sanierungszuweisungen und schützen damit das verfassungsrechtliche System des Finanzausgleichs vor Nivellierung und Privilegierung. Darüber hinaus konkretisieren sie den Maßstab für die verfassungsgerichtliche Kontrolle der politischen Entscheidung.

\section{Grenzen von Sanierungszuweisungen}

Das „Berlin-Urteil“ des BVerfG präzisiert nicht nur die Voraussetzungen von Sanierungszuweisungen im geltenden Finanzverfassungsrecht, sondern hebt zugleich auch deren Grenzen hervor. Abgesehen davon, dass als Sonderbedarfszuweisungen geleistete Sanierungshilfen ihre Grenzen stets in den rechtfertigenden Gründen finden, sind ein Nivellierungsverbot und ein Gleichbehandlungsgebot $\mathrm{zu}$ beachten. ${ }^{26}$

\section{a) Nivellierungsverbot}

Nach dem Nivellierungsverbot ist es dem Bund untersagt, mithilfe von Bundesergänzungszuweisungen nach Art.107 Abs. 3 S. 2 GG die Finanzkraftreihenfolge unter den Geberländern zu verändern und den leistungsschwachen Ländern eine überdurchschnittliche Finanzkraft $\mathrm{zu}$ verschaffen. ${ }^{27}$ Selbst wenn also ausnahmsweise einmal ein bundesstaatli-

$22 B \operatorname{Verf} G E$ 79, 311 (344f.).

$23 B \operatorname{VerfGE} 116,327,391$.

24 So die Überlegung in $B \operatorname{VerfGE} 79,311$ (344f.).

25 VerfGH DVBl. 2004, 308 ff.; dazu Höfling, in: FS für von Arnim, 2004, 259 ff.; Jochimsen DÖV 2004, 511 ff.; Kerber DÖV 2004, 691 ff.; Pestalozza LKV 2004, 63 ff.; Rossi DVl 2005, 269 ff.; Waldhoff NVwZ 2004, $1062 \mathrm{ff}$.

$26 B \operatorname{VerfGE} 116,327,391$.

$27 B \operatorname{VerfGE} 116,327,381 \mathrm{f}$ cher Notstand vorliegen sollte und deshalb Sonderergänzungszuweisungen nach Art.107 Abs.3 S.2 GG zur Sanierung des Haushalts eines Landes zulässig und geboten sind, so darf dieses Land dadurch keinen relativen Vorteil gegenüber den anderen Ländern erhalten. Während also der bundesstaatliche Notstand nur über das „Ob“ von Sanierungszuweisungen entscheidet, wird deren maximale Höhe durch das Nivellierungsverbot begrenzt: Sanierungszuweisungen sind auch in einem bundesstaatlichen Notstand nur bis zu einer Höhe zulässig, die die relative Finanzkraft des Landes im Verhältnis zu den anderen Ländern nicht verändert. Das Nivellierungsverbot, das in Ansätzen auch schon für die anderen Stufen des Finanzausgleichssystems gilt, ${ }^{28}$ ist somit ebenso konsequente Folge der Charakterisierung von Sanierungszuweisungen als Fremdkörper im geltenden Finanzausgleichssystem wie notwendige Ergänzung des strengen Ultima-ratio-Prinzips.

\section{b) Gleichbehandlungsgebot}

Das föderative Gebot der Gleichbehandlung aller Länder durch den Bund folgt unmittelbar aus dem Bundesstaatsprinzip und dem allgemeinen Gleichheitssatz. ${ }^{29}$ Wie stets bei Gleichheitsfragen, lässt sich das Gleichbehandlungsgebot also auch als Diskriminierungs- bzw. Privilegierungsverbot verstehen, wobei letzteres die praktische Bedeutung fast besser verdeutlicht, weil es bei der Zuweisung von zusätzlichen Geldern primär um eine Besserstellung eines Landes geht, die als (automatische) Nebenfolge die Schlechterstellung anderer Länder nach sich zieht. Zum besseren Verständnis dieses Verbots lässt sich an die Rechtsprechung des BVerfG zu den Verwaltungsvereinbarungen nach Art.104a Abs. 4 GG a.F. anknüpfen, in der es hervorgehoben hat, dass der Bund, will er nicht seine Pflicht zu bundesfreundlichem Verhalten verletzen, eine solche Vereinbarung nur mit allen gleichermaßen betroffenen Ländern abschließen darf, wobei die Länder dem Bund als gleichberechtigte Partner gegenüberstehen. Die Gültigkeit der Vereinbarung hängt deshalb grundsätzlich von der Zustimmung aller betroffenen Länder ab. 30

Aus dieser Rechtsprechung lässt sich in Bezug auf Sanierungszuweisungen zweierlei ableiten: In prozeduraler Hinsicht ist es dem Bund verwehrt, einem Land besondere finanzielle Hilfen zur Verfügung zu stellen, ohne die anderen Länder an der Entscheidung zu beteiligen. Vor allem aber kann in materieller Hinsicht dem föderalen Gleichbehandlungsgebot letztlich nur durch eine antizipierte Maßstabsbildung Rechnung getragen werden, an der alle Glieder beteiligt sind. Bund und Länder sind insofern aufgefordert, einheitliche abstrakte Vorgaben für die ausnahmsweise Gewährung von Sanierungszuweisungen zu normieren, auf deren Grundlage dann in jedem Einzelfall entschieden werden kann und muss, ob und in welcher Höhe konkrete Sanierungszuweisungen gezahlt werden. ${ }^{31} \mathrm{Bis} \mathrm{zu}$ einer solchen gesetzgeberischen Maßnahme bindet das föderale Gleichbehandlungsgebot vor allem das $B \operatorname{Verf} G$, das sich dieser Bindung ausweislich der bereits wiedergegebenen Worte des Vorsitzenden des 2. Senats durchaus bewusst ist. Er hat mit Blick auf die anhängigen Anträge Bremens und des Saarlands

\footnotetext{
28 Vgl. schon $B \operatorname{VerfGE~1,~} 117$ (131); 72, 330 (398); 101, 158 (222); deutlich auch $B \operatorname{VerfGE} 116,327,380$ : „kein Systemwechsel“.

29 Vgl. $B \operatorname{VerfGE} 72,330$ (404).

$30 B \operatorname{VerfGE} 41,291$ (308 u. Ls. 2).

31 Zur Notwendigkeit eines solchen zweistufigen Verfahrens vgl. Rossil Schuppert ZPR 2006, 8 (9).
} 
deutlich gemacht, dass der Senat diese Anträge an dem im Berlin-Urteil gefundenen Maßstab entscheiden wird.

\section{Konsequenzen für hilfebegehrende Bundesländer}

Wegen dieser Maßstabsbildung zeitigt das Berlin-Urteil für alle hilfebegehrende Bundesländer Konsequenzen, wobei es naturgemäß für Berlin besondere Implikationen entfaltet.

\section{Allgemeines}

Nach dem Berlin-Urteil bleiben den Ländern, die sich in einer extremen Haushaltsnotlage wähnen, theoretisch nur zwei Möglichkeiten: Sie können strikt konsolidierend ihre Verschuldung reduzieren oder eher konfligierend in den bundesstaatlichen Notstand steuern. Die Entscheidung zwischen diesen Möglichkeiten hängt auch von den unterschiedlichen Mentalitäten der politischen Akteure in den einzelnen Ländern ab: Brandenburg etwa will Geberland werden, Berlin dagegen erwartet noch immer Hilfen als Notlagenland. Jenseits solcher grundsätzlichen politischen Erwägungen sind die finanziellen Spielräume der notleidenden Länder schwer zu beurteilen. Einerseits darf die Schwierigkeit der stark verschuldeten Länder, sich aus eigener Kraft aus der Verschuldung zu ziehen, nicht unterschätzt werden. Für Berlin etwa gibt es Berechnungen, dass dies ohne fremde Hilfe in absehbarer Zeit nicht möglich ist. ${ }^{32}$ Andererseits haben unerwartete Steuermehreinnahmen die Haushaltslage auch der notleidenden Länder erheblich verbessert. Letztlich hoffen deshalb derzeit nur noch das Saarland und Bremen auf Sonderzuweisungen des Bundes. Ihnen bleiben zur Plausibilisierung ihrer entsprechenden Normenkontrollanträge an das $B$ VerfG nur die Möglichkeiten, entweder einen bundesstaatlichen Notstand darzulegen und zu begründen oder aber bestimme Sonderlasten geltend zu machen. Das BVerfG hat nämlich deutlich hervorgehoben, dass Sonderbedarfszuweisungen nur für „Sonderlasten auf Grund (verfassungsrechtlich) vorgegebener Aufgaben außerhalb des autonomen landespolitischen Gestaltungsraums“ in Betracht kommen. Gekennzeichnet sind solche Sonderlasten dadurch, dass sie „nicht in ähnlicher Höhe oder überhaupt nicht in allen Ländern zu leisten sind, sondern nur in einem Land oder einer Gruppe von Ländern anfallen und nicht auf den vorangegangen Stufen des Finanzausgleichs zu berücksichtigen waren. “33

\section{Konsequenzen für Berlin}

Für Berlin hat das Urteil zur unmittelbaren Konsequenz, dass das Land keinerlei Anspruch auf weitere Hilfen des Bundes zur Finanzierung seines Haushalts hat. Zugleich verändert es aber auch die rechtlichen Rahmenbedingungen (a) für die politischen Handlungsoptionen des Landes (b).

\section{a) Rechtliche Rahmenbedingungen}

In rechtlicher Hinsicht wirft das Urteil für Berlin die abstrakte Frage auf, wie sich die Entscheidung des BVerfG zur zeitlich vorhergehenden Entscheidung des $L V \operatorname{erf} G$ verhält. Der VerfGH Berlin hat in seiner Entscheidung vom 31.10. 2003 zwar den Doppelhaushalt für die Jahre 2002/2003 we-

32 Fiedler u.a. (Fn. 20), S. $218 \mathrm{ff}$.

33 BVerfGE 116, 327, 384. gen unzulässiger Überschreitung der landesverfassungsrechtlichen Verschuldungsgrenze für verfassungswidrig und nichtig erklärt, zugleich aber im Wege der Rechtsfortbildung eine überverfassungsrechtliche Ausnahme kreiert. Danach soll ein Überschreiten der landesverfassungsrechtlichen Begrenzung der Kreditfinanzierung auf die Investitionshöhe ausnahmsweise zulässig sein, wenn und soweit sich das Land in einer extremen Haushaltsnotlage befindet und die Überschreitung erforderlich ist, um „bundesrechtlich festgelegte sowie auf landesverfassungsrechtlichen Vorgaben beruhende Ausgabenverpflichtungen " 34 zu erfüllen. Der Berliner Haushaltsgesetzgeber hat sich bei den weiteren Haushaltsplänen für die Jahre 2004/2005 und 2006/2007 auf diese überverfassungsrechtliche Ausnahme gestützt, um die abermalige Überschreitung der verfassungsrechtlich zulässigen Verschuldung zu rechtfertigen.

Das $B \operatorname{VerfG}$ hat sich zu dieser in seinen Konsequenzen umstrittenen Entscheidung des VGH Berlin ${ }^{35}$ nicht ausdrücklich geäußert, sondern die Zulässigkeit einer über(landes)verfassungsrechtlichen Ausnahme zurückhaltend der Verfassungsautonomie des Landes überlassen. Indirekt lassen sich dem Berlin-Urteil des BVerfG gleichwohl Bezugspunkte zur Entscheidung des VerfGH Berlin entnehmen.

Auf der einen Seite greift das BVerfG die vom VerfGH vorgenommene Unterscheidung zwischen obligatorischen und nichtobligatorischen Ausgaben auf, wenn es klarstellt, dass Sonderbedarfszuweisungen nach Art.107 Abs.3 S.2 GG nur für „Sonderlasten auf Grund (verfassungsrechtlich) vorgegebener Aufgaben außerhalb des autonomen landespolitischen Gestaltungsraums “ geleistet werden dürfen. ${ }^{36}$

Auf der anderen Seite ist nicht zu verkennen, dass die bloße (behauptete) Notwendigkeit, eine überverfassungsrechtliche Ausnahme in Anspruch nehmen zu müssen, offensichtlich nicht genügt, um einen „bundesstaatlichen Notstand“ zu begründen. Mag also die vom VerfGH Berlin nicht nur geduldete, sondern sogar geschaffene Möglichkeit, die verfassungsrechtlichen Verschuldungsgrenzen unter bestimmten Vorraussetzungen zu überschreiten, ohne verfassungswidrig zu handeln, auch grundsätzlich eine Notlage indizieren, so ist dies nicht oder jedenfalls nicht automatisch zugleich auch ein „bundesstaatlicher Notstand“. Erneut zeigt sich, dass der Begriff „bundesstaatlicher Notstand“ nicht primär auf die Situation im betroffenen Land rekurriert, sondern vor allem die Auswirkungen der angespannten Haushaltslage eines Landes auf den gesamten Bundesstaat im Blick hat. Dementsprechend ist es für die Beurteilung der Frage, ob ein „bundesstaatlicher Notstand“ vorliegt oder nicht, irrelevant oder zumindest doch nicht entscheidend, ob und mit welchen Konsequenzen Landesverfassungsrecht verletzt wird oder sogar wegen der vermeintlichen Notlage verletzt werden muss. Die vom VerfGH Berlin in einem obiter dictum entwickelte Figur der überverfassungsrechtlichen Ausnahme vom grundsätzlichen Verschuldungsverbot wird insoweit ohne Bedeutung bleiben.

Unabhängig von dem abstrakten Verhältnis zwischen den grundgesetzlichen Voraussetzungen, unter denen Sanierungszuweisungen des Bundes an ein Bundesland zulässig und geboten sind, und den landesverfassungsrechtlichen Voraussetzungen, unter denen die Verschuldung ausnahmsweise die verfassungsrechtlich vorgesehene Begrenzung überschreiten darf, hat die Entscheidung des BVerfG ganz

34 VerfGH Berlin DVB1. 2004, 308 (312).

35 Vgl. Rossi DVBl. 2005, 269 (272); Höfling DVBl. 2006, 934 (938); Waldhoff NVwZ 2004, 1062 (1064).

$36 B \operatorname{VerfGE} 116,327,384$. 
konkrete Auswirkungen jedenfalls auf den aktuellen Haushaltsplan des Landes Berlin. Wenn nämlich das BVerfG deutlich festhält: Das Land Berlin „,befindet sich nicht in einer extremen Haushaltsnotlage“, 37 dann ist es diesem verwehrt, sich für die Überschreitung der landesverfassungsrechtlichen Kreditbegrenzungsregel auf die vom VerfGH begründete überverfassungsrechtliche Ausnahme zu berufen. Deren Voraussetzungen liegen schlicht nicht vor. Der Berliner Doppelhaushalt für die Jahre 2006/2007 ist insofern verfassungswidrig, weil und soweit die Verschuldung für die einzelnen Haushaltsjahre über den entsprechenden Investitionshöhen liegt. Ob diese Verfassungswidrigkeit jedenfalls in Bezug auf das Haushaltsjahr 2007 noch durch einen korrigierenden Nachtragshaushaltsplan vermieden werden kann, soll an dieser Stelle nicht entschieden werden, steht aber wohl auch nicht auf der politischen Agenda der rot-roten Mehrheit im Abgeordnetenhaus.

\section{b) Politische Handlungsoptionen}

Das Fehlen eines Rechtsanspruchs bedeutet freilich nicht, dass Berlin nicht von den anderen Körperschaften des Bundes finanzielle Hilfe bekommen darf. Es ist insofern allerdings ausschließlich auf die freiwillige Zahlungsbereitschaft in Bund und Ländern angewiesen und müsste seine Politik und Diplomatie entsprechend ausrichten. Als glaubhafter Verhandlungspartner wird Berlin dabei sicherlich erst dann ernst genommen, wenn es seine - vom Senator für Finanzen nach Verkündung des Berlin-Urteils schnell erkannten und verkündeten - Sparpotentiale voll ausnutzt. Vor dem Hintergrund, dass Berlin sich selbst als arm aber sexy beurteilt, wird es sich insbesondere auch die Frage gefallen lassen müssen, ob sich aus seiner Attraktivität nicht mehr Kapital schlagen lässt.

Unabhängig von diesen allgemeinen finanz- und haushaltspolitischen Überlegungen ist zu betonen, dass Berlins Politik in Bezug auf die anderen Länder und den Bund nicht unmittelbar auf weitere Zahlungen gerichtet sein muss. Vielmehr könnte sich eine kluge und nachhaltige Politik auch darauf richten, politische Initiativen, etwa über den Bundesrat, für die Schaffung eines bundesweiten Haushaltsnotlagenregimes zu ergreifen, von denen dann auch Berlin profitieren könnte. Zusammen mit den anderen Bundesländern, die eine extreme Haushaltsnotlage für sich reklamieren, jedenfalls aber zusammen mit den anderen Nehmerländern stellt Berlin hier eine beachtliche Mehrheit dar, derer es sich offensichtlich nicht bewusst ist oder die es aus parteipolitischen Erwägungen nicht nutzen will.

\section{Erlass eines Haushaltsnotlagenregimes}

Mit seiner Entscheidung, Berlin einen Anspruch auf Sanierungszuweisungen $\mathrm{zu}$ versagen, hat das BVerfG die Entschuldungsverantwortung nicht nur zurück in die Politik der Berliner Provinz, sondern auch in die Politik der Berliner Republik gespielt. Bund und Länder sind angesprochen, ein Haushaltsnotlagenregime zu erlassen.

\section{Kein verbindlicher Handlungsauftrag}

Zwar hat sich das $B \operatorname{VerfG}$ primär zurückhaltend auf seine reagierend-kontrollierende Funktion beschränkt und nicht agierend-initiierend auf den Gesetzgeber eingewirkt. Es hat

$37 B \operatorname{VerfGE} 116,327,394$. dementsprechend den konkreten Antrag Berlins auf zusätzliche Bundeshilfen zurückgewiesen, ohne zugleich den politischen Akteuren auf Bundes- und Landesebene vorzuschreiben, wie mit aktuellen und künftigen Haushaltsnotlagen umzugehen ist.

So sehr diese Zurückhaltung im Interesse eines weitgehenden Gestaltungsspielraums der Politik auch grundsätzlich zu begrüßen ist, so sehr ist dies zu bedauern, wo es der Politik, ihren Institutionen und Akteuren aufgrund ihren eigenen Gesetzlichkeiten an Kraft fehlt, grundsätzliche Probleme der föderalen Beziehungen aus eigenem Antrieb zu lösen. Aus diesem Grunde wäre es wünschenswert gewesen, dass das $B \operatorname{VerfG}$ entweder einen ausdrücklichen Impuls, vielleicht gar einen konkreten Handlungsauftrag 38 für den Umgang mit der Haushaltsnotlage in Berlin im Besonderen und Haushaltsnotlagen in anderen Ländern im Allgemeinen gegeben hätte oder es - quasi in antizipierter Anwendung das Grundkonzept eines solchen Haushaltsnotlagenregimes am Beispiel der Bewältigung der Haushaltsnotlage Berlins dargelegt hätte.

\section{Dezidierte Handlungsaufforderung}

Lässt sich der Entscheidung ein solcher Handlungsauftrag explizit auch nicht entnehmen, weist das $B \operatorname{Verf} G$ doch deutlich und mehrmals auf die Notwendigkeit hin, Regelungen über die Behandlung, möglichst auch über die Vermeidung von Haushaltsnotlagen in den Bundesländern zu schaffen, und spricht insofern dezidiert den Bundesgesetzgeber an. Ausgehend von dem Befund, dass „das geltende positive Recht [...] nur unzureichend auf die Bewältigung auftretender extremer Haushaltsnotlagen eingestellt“ ist ${ }^{39}$ und „das Instrumentarium des geltenden Finanzausgleichsrechts" mit „Aufgaben der Haushaltssanierung einzelner Länder [...] überfordert wird“ "40, konstatiert das $B \operatorname{Verf} G$ als „gravierende Schwäche des geltenden Rechts [...], dass es an den notwendigen verfahrensrechtlich wie auch inhaltlich handlungsleitenden Regelungen zum Umgang mit potentiellen und aktuellen Sanierungsfällen im Bundesstaat fehlt" ${ }^{\text {41 }}$, erinnert sodann an die bereits in der Entscheidung vom 27. 5. 1992 ausgesprochene Mahnung, es sei „zuvörderst nötig und besonders dringlich [...], Bund und Länder gemeinsam treffende Verpflichtungen und Verfahrensregelungen festzulegen, die der Entstehung einer Haushaltsnotlage entgegenwirken und zum Abbau einer eingetretenen Haushaltsnotlage beizutragen geeignet sind“, und bekräftigt diese Mahnung mit den Worten: „Das Bundesstaatsprinzip macht solche Bestrebungen angesichts der gegenwärtig defizitären Rechtslage erforderlich.“ $" 42$

\section{Handlungsrahmen}

Der Bundesgesetzgeber ist also gehalten (wenn auch nicht verpflichtet), ein Haushaltsnotlagenregime $\mathrm{zu}$ entwickeln. Ist er in der konkreten Ausgestaltung eines solchen Notlagenregimes auch weitgehend frei, so sind die grundsätzliche Leitmaxime und die äußeren verfassungsrechtlichen Grenzen eines solchen Notlagenregimes freilich vom $B \operatorname{Verf} G$ besonders hervorgehoben worden: Leitmaxime ist die Eigen-

38 Zum Gesetzgebungsauftrag, ein Maßstäbegesetz zu erlassen, vgl.

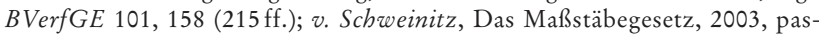
sim.

39 BVerfGE 116, 327, 377.

$40 B \operatorname{VerfGE} 116,327,392$

41 BVerfGE 116, 327, 393.

42 BVerfGE 116, 327, 394. 
ständigkeit und Eigenverantwortlichkeit der Länder, Grenzen ergeben sich insbesondere aus dem (derzeitigen) System des verfassungsrechtlichen Länderfinanzausgleichs sowie aus dem Verfassungsgrundsatz der Statusgleichheit der Länder. Damit ist ein Handlungsrahmen vorgegeben worden, der aus einem flexiblen und einem starren Element besteht. Denn während - etwa im Zuge der Föderalismusreform II mit den Finanzbeziehungen zwischen dem Bund und den Ländern auch das System des Länderfinanzausgleichs und damit der Rahmen für Sanierungszuweisungen im Notlagenfall modifiziert werden kann, muss sich jedes veränderte oder neu geschaffene System am Grundsatz der Statusgleichheit der Länder messen lassen. Dieser Grundsatz nämlich ist der bundesstaatlichen Ordnung derart immanent, dass er über Art. 79 Abs.3 GG sogar der Dispositionsfreiheit des verfassungsändernden Gesetzgebers entzogen ist. ${ }^{43}$

\section{Handlungsmöglichkeiten}

Dem Bundesgesetzgeber stehen so viele Handlungsmöglichkeiten zur Verfügung, dass sie hier nicht alle vorgestellt werden können. Hervorzuheben ist bei grundsätzlicher Betrachtung allerdings, dass er sich entweder im skizzierten bestehenden Handlungsrahmen bewegen oder aber diesen - in den Grenzen des Art. 79 Abs. 3 GG - selbst modifizieren kann. Angesprochen ist also nicht nur der einfache, sondern auch der verfassungsändernde Gesetzgeber, der sich mit der Föderalismusreform II ja ohnehin das politische Ziel gesetzt hat, die Finanzbeziehungen zwischen dem Bund und den Ländern neu zu ordnen.

Von den zahlreichen denkbaren und zum Teil auch schon gedachten Handlungsmöglichkeiten ${ }^{44}$ sollen im Folgenden drei skizziert werden, die wegen der jeweils zu nehmenden rechtlichen und politischen Hürden als kleine, mittlere und große Lösung bezeichnet werden können. Sämtliche dieser Möglichkeiten können und sollten miteinander kombiniert werden.

\section{a) Subjektivierung der Verschuldungsgrenzen}

Eine erste Möglichkeit besteht in der Entpolitisierung und Wiederverrechtlichung, möglicherweise gar der Subjektivierung der Verschuldungsgrenzen. Zwar normieren alle Landesverfassungen - zumeist in Anlehnung an die Bestimmung des Art. 115 GG - Grenzen der Staatsverschuldung. ${ }^{45}$ Doch in der Praxis haben diese verfassungsrechtlichen Verschuldungsgrenzen durch ihre ständige Missachtung erheblich an Verbindlichkeit verloren, ${ }^{46}$ wie an der zunehmenden Schamlosigkeit deutlich wird, mit der sich die politischen Akteure über diese Vorgaben hinwegsetzen. Dass die Missachtung der verfassungsrechtlichen Verschuldungsgrenzen in der Praxis meist ohne Konsequenzen bleibt, liegt in erster Linie an fehlenden Klägern. Aus (partei-)politischen Gründen sehen die Oppositionsfraktionen häufig davon ab, die Überschreitung der verfassungsrechtlich zulässigen Kreditaufnahme vom jeweiligen Verfassungsgericht feststellen zu lassen, um nicht später bei einer etwaigen Regierungsbeteiligung an selber Elle gemessen zu werden. Außerdem lässt sich eine konsolidierungsorientierte Haushaltspolitik dem Wähler nach wie vor schlechter vermitteln als eine ausgabefreudige Sachpolitik.

43 Vgl. dazu Boysen, Gleichheit im Bundesstaat, 2005, S. 200 f. u. passim. 44 Vgl. die Beiträge von Waldhoff und Hey auf der Staatsrechtslehrertagung 2006 in Rostock; Gröpl, Haushaltsrecht und Reform, 2001.

45 Vgl. Kloepfer/Rossi VerwArch 94 (2003), 319 (323 ff.)

46 Vgl. jüngst Höfling DVBl. 2006, $934 \mathrm{ff}$.
Materielle Verschuldungsregeln mit „Biss“47 ließen sich vor diesem Hintergrund bspw. schon durch prozedurale $\mathrm{Maßnahmen} \mathrm{erreichen.} \mathrm{Bereits} \mathrm{de} \mathrm{constitutione} \mathrm{lata} \mathrm{ist} \mathrm{zu}$ überlegen, ob nicht jede Landesregierung befugt ist, den Haushaltsplan eines anderen Landes mit der Behauptung vom $B V \operatorname{verf} G$ überprüfen zu lassen, die Überschreitung der zulässigen Kreditobergrenze erschwere dem Bund die Einhaltung seiner Verpflichtung gegenüber der Europäischen Union und verletzte daher das bundesstaatliche Rücksichtnahmegebot. ${ }^{48}$ Freilich werden die Landesregierungen aus falsch verstandenem parteipolitischen Kalkül von solchen Klagemöglichkeiten regelmäßig keinen Gebrauch machen.

Notwendig wird es deshalb sei, de lege ferenda den Kreis der Klagebefugten zu erweitern. Behutsam könnte man etwa den sachverständigen und unabhängigen Rechnungshöfen das Recht zubilligen, bei Missachtung der verfassungsrechtlichen Verschuldungsgrenzen das jeweilige Verfassungsgericht anzurufen. ${ }^{49}$ Etwas weitergehend könnten auch entsprechende Verbandsklagerechte statuiert werden, von denen etwa der Bund der Steuerzahler Gebrauch machen könnte. Denkbar ist schließlich auch eine radikale Ausweitung des Klägerkreises auf jeden Einzelnen. Eine solche Subjektivierung der Verschuldungsgrenzen könnte unter Berufung auf die Schutznormlehre möglicherweise schon durch die Rechtsprechung der Verfassungsgerichte erfolgen. Denn dass die verfassungsrechtlichen Verschuldungsgrenzen jedenfalls auch zum Schutze der Einzelnen fungieren, lässt sich unter Rückgriff auf den Nachhaltigkeitsgrundsatz und den Grundgedanken der Generationengerechtigkeit durchaus begründen. Zwar ist das Nachhaltigkeitsprinzip explizit nur in Art. 20a GG und somit inhaltlich beschränkt auf den Umweltschutz normiert, doch lässt es sich ebenso wie die Generationengerechtigkeit letztlich auch aus dem Demokratieprinzip ableiten. Als Grundregel der Nachhaltigkeit hat die Bundesregierung jedenfalls jüngst formuliert: „Jede Generation muss ihre Aufgaben selbst lösen und darf sie nicht den kommenden Generationen aufbürden. Dagegen verstößt, wer eine Wirtschafts- und Sozialpolitik auf Pump macht und die steigenden Staatsschulden den künftigen Generationen hinterlässt. "50 Ergänzt man diese Regel differenzierend auf die Besonderheiten eines Bundesstaates, wird deutlich, dass es weder in zeitlicher noch in räumlicher Hinsicht zulässig ist, selbst verantwortete Schulden auf nachfolgende Generationen oder andere Personenverbände zu übertragen.

Die Entscheidung des BVerfG enthält einen ersten Hinweis auf ein derartig subjektiv-rechtliches Verständnis nicht nur der Haushalts-, sondern auch der Finanzausgleichsvorschriften, denn es arbeitet heraus, dass „die verfassungsgemäße Wahrnehmung objektiver Organkompetenzen im Interesse (bundes-)staatlicher Aufgabenerfüllung gegenüber den Bürgern“ erfolgt. Zugleich betont es, dass die bundesstaatliche Einstandspflicht im Notstand nicht nur im Interesse der Bürger des jeweiligen Landes, sondern im Interesse der Bundesbürger erfolgt..$^{51} \mathrm{Ob}$ es vor diesem Hintergrund eine mit der Behauptung erhobene Verfassungsbeschwerde, durch die übermäßige Verschuldung in Art. 2 Abs. 1 GG verletzt zu sein, annehmen würde, muss gleichwohl bezweifelt werden. Doch festzuhalten ist mit Ihering, dass zugleich das

47 So die Forderung der Rechnungshöfe, zitiert nach Zimmermann Wirtschaftsdienst 2006, 391 (397).

48 Kloepfer/Rossi VerwArch 94 (2003), 319 (336),

49 Vgl. Rossi DVBl. 2005, 269 (275 f.)

50 Bundesregierung, Perspektiven für Deutschland, Unsere Strategie für eine nachhaltige Entwicklung, 2002, S. 5.

$51 B \operatorname{VerfGE} 116,327,391 \mathrm{f}$ 
Recht schützt, wer sein Recht schützt. ${ }^{52}$ Ebenso wie deshalb die Mobilisierung der Bürger für die Durchsetzung des Rechts $^{53} \mathrm{zu}$ einem wesentlichen Faktor für die Beachtung und einheitliche Anwendung der europarechtlichen Bestimmungen geworden ist, könnte eine Subjektivierung der Verschuldungsgrenzen $\mathrm{zu}$ einem wirksamen Instrument ihrer Einhaltung führen.

\section{b) Erlass eines Haushaltsnotlagenregimes}

Die bloße Ausweitung der Klagebefugnisse hat für sich alleine genommen freilich zwei Nachteile: Zum einen kann eine Beachtung der verfassungsrechtlichen Verschuldungsgrenzen extreme Haushaltsnotlagen nur für die Zukunft und auch dann nur mit hoher Wahrscheinlichkeit ausschließen, bietet aber keine Handhabe für bereits oder gleichwohl eingetretene extreme Haushaltsnotlagen. Zum anderen weist die Ausweitung der Klagebefugnisse stets nur den Weg zu den Verfassungsgerichten, die aufgrund ihrer Funktion und Besetzung nicht als optimale Entscheidungsorgane für die Bewältigung von Haushaltskrisen im Bundesstaat angesehen werden können.

Insofern scheint es dringend geboten, ein Haushaltsnotlagenregime zu schaffen, das auch den Umgang mit eingetretenen Haushaltsnotlagen umfasst und einen entsprechenden Ausgang weist. Der wissenschaftliche Beirat beim Bundesfinanzministerium hat einen solchen Vorschlag unterbreitet, die Hertie-School of Governance eine entsprechende Studie vorgelegt. ${ }^{54}$ Diese Studie zeigt eine Möglichkeit auf, wie Haushaltsnotlagen im Bundesstaat nach Möglichkeit verhindert, gegebenenfalls festgestellt und dann auch beseitigt werden können. Die Grundidee der konditionierten Bewilligung, der Unterstützung Zug-um-Zug gegen bestimmte Sanierungsmaßnahmen, bleibt insofern als eine verfassungsrechtlich zulässige, finanzwissenschaftlich sinnvolle und politisch zu realisierende Möglichkeit eine der wichtigsten Handlungsalternativen solcher Überlegungen, die freilich durch andere Maßnahmen, etwa durch die Schaffung stren-

52 v. Ihering, Der Kampf ums Recht, 13. Aufl. 1897, S. 47 f.

53 So der Titel der Arbeit von Masing, 1997; vgl. ders., in: HoffmannRiem/Schmidt-Aßmann/Voßkuble (Hrsg.), Grundlagen des Verwaltungsrechts, Bd. I, 2006, S. $391 \mathrm{ff}$.

54 Fiedler u.a. (Fn. 20), passim. gerer und verbindlicherer Verschuldungsgrenzen, flankiert werden könnte. Das zur Umsetzung dieser Grundidee von der Studie vorgeschlagene zweistufige Modell, konkrete Sanierungsverträge nur auf der Grundlage eines abstrakten Haushaltsnotlagengesetzes zu erlassen, eignet sich in besonderer Weise zur Einhaltung des Nivellierungsverbots und wird vor allem auch dem Grundsatz der föderalen Gleichbehandlung gerecht.

\section{c) Neugliederung des Bundesgebiets}

Das BVerfG spricht im Berlin-Urteil schließlich auch die Möglichkeit einer Neugliederung des Bundesgebiets an. Während es allerdings in einer seiner ersten Entscheidungen noch der Auffassung war, dass der Finanzausgleich „mit Rücksicht auf Art.29 GG nicht zu dem Ergebnis führen [darf], lebensunfähige Länder künstlich am Leben zu erhalten," 55 und in seiner Entscheidung zu den Finanzhilfen für Bremen und das Saarland relativ neutral mit dem Wortlaut des Art. 29 GG auf dessen Zweck hingewiesen hat, zu gewährleisten, dass die Länder nach Größe und Leistungsfähigkeit die ihnen obliegenden Aufgaben wirksam erfüllen, ${ }^{56}$ scheint es nunmehr vor allem den Bund und die Geberländer anzusprechen: „Solange der verfassungsrechtlich eröffnete Weg einer Neugliederung des Bundesgebiets nicht beschritten worden ist, ist es bundesstaatliches Gebot, die Existenz des Not leidenden Landes als eines handlungsfähigen Adressaten verfassungsrechtlicher Pflichten und als eines Trägers verfassungsrechtlicher Aufgaben auch finanziell zu gewährleisten. "57 Vor dem Hintergrund, dass die Entscheidung durchgängig und auch im Ergebnis die Eigenverantwortung der Länder betont und Hilfsansprüche unter praktisch kaum $\mathrm{zu}$ erfüllende Voraussetzungen stellt, scheint dieser an entsprechend unwahrscheinliche Hilfspflichten anknüpfende Druck nicht geeignet, eine Neugliederung des Bundesgebiets anzustoßen. Sie ist derzeit jedenfalls nicht Gegenstand der föderalen Reformüberlegungen und deshalb auch keine realistische politische Option.

$55 B \operatorname{Verf} G E$ 1, 117 (134 u. Ls. 1).

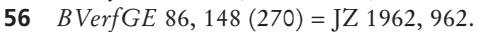

$57 B \operatorname{VerfGE} 116,327,386 f$.
Ulrich Karpen: Gesetzgebungslehre - neu evaluiert. Legistics freshly evaluated. - Baden-Baden: Nomos Verlag, 2006. $201 \mathrm{~S}$.; Pb.: 44.- €. ISBN 3-8329-1980-5.

In diesem Band legt Ulrich Karpen, emeritierter Professor der Universität Hamburg und langjähriger Vorsitzender der Deutschen Gesellschaft für Gesetzgebung (DGG) und der European Association of Legislation (EAL), eine Reihe von Aufsätzen vor, die teils schon publiziert worden sind, teils noch nicht. Der Band erscheint 20 Jahre nach der Gründung der „Zeitschrift für Gesetzgebung“, einer Vierteljahresschrift für staatliche und kommunale Gesetzgebung, und weist so auf den Aufschwung eines Wissenschaftszweigs hin, der seit 1986 sehr viel mehr Interesse gefunden und Autoren angezogen hat als in den Jahrzehnten, ja Jahrhunderten davor, die sich mit guter Gesetzgebung befasst haben. Das hat mehrere Gründe: Beschleunigung der Lebensverhältnisse, zunehmende Normenflut und Mehrebenenregierung, Entwicklung einer modernen Verwaltungswissenschaft sowie Steuerungswissenschaft in mehreren Zweigen von Sozial- und Rechtswissenschaft, Privatisierungstendenzen, höhere Ansprüche sowohl an generelle wie individuelle Angemessenheit von Rechtsregelungen.
Karpen spricht die neuere Entwicklung der Gesetzgebungslehre im deutschsprachigen Raum an und geht auf alle genannten Punkte mehrfach ein. Ein Teil der Kapitel hat Übersichtscharakter. „Zum Stand der Gesetzgebungswissenschaft in Europa" heißt Kap.1, das auf Englisch in Kap. 6 wiederholt wird. Kap. 5 befaßt sich mit „Gesetzesfolgenabschätzung in der Europäischen Union“ und wird in Kap. 10 ebenfalls großteils in englischer Übersetzung noch einmal abgedruckt. In diesen Beiträgen geht der Autor nicht nur auf die Lage in Deutschland und in der EU, sondern auch in vielen Mitgliedstaaten der EU ein. Konkreter sind Kap. 2 „Weniger und bessere Gesetze - Voraussetzung der Entbürokratisierung“ und Kap. 3 „Eine Legislaturperiode des Deutschen Bundestags wird besichtigt (2002-2005)“. Als besonders gut gelungenes Beispiel von Verfassungsgesetzgebung stellt Karpen „Das Grundgesetz als Exportartikel“ (Kap.4) vor. Untertitel: „Warum sich andere Länder für das Grundgesetz interessieren“. Die Analyse ist kurz und schlüssig, an einer Stelle freilich auch etwas kurzschlüssig. Das Interesse vieler mittel- und osteuropäischer sowie lateinamerikanischer Länder am Grundgesetz und $B \operatorname{Verf} G$ bezieht sich nicht nur allgemein auf das von Karlsruhe entwickelte Grundrechtsdenken, sondern besonders auf die objektivrechtliche und sozialstaatliche Dimension der Grundrechte, 
